\title{
The quasi-reversibility regularization method for a Cauchy problem for the Laplace equation
}

\author{
Juanjuan Pu, Xiangtuan Xiong
}

\begin{abstract}
In this paper, the Cauchy problem for the homogeneous and nonhomogeneous Laplace equation in a strip domain is discussed. Where the Cauchy data at $x=0$ is given, and the solution is found in the interval $(0,1)$. The corresponding error estimation is obtained by a new quasi-reversibility regularization method with a appropriate regularization parameter selection.
\end{abstract}

Index Terms - Ill-posed problem; the Cauchy problem for the Laplace equation; Quasi-reversibility regularization; Error estimate.

\section{INTRODUCTION}

The Cauchy problem for the Laplace equation arises from many physical and engineering problems such as plasma physics [1], cardiology [2] and seismology [3] all require the solution of a Cauchy problem for the Laplace equation. For example, certain problems related to the search for mineral resources, which involve interpretation of the earth's gravitational and magnetic fields, are equivalent to the Cauchy problem for the Laplace equation. The continuation of the gravitational potential observed on the surface of the earth in a direction away from the sources of the field is again such a problem.

It is well-know that, the Cauchy problem for the Laplace equation is severely ill-posed. A small change in the Cauchy data may result in a dramatic change in the solution. Usually, there are no solution that satisfy Cauchy date, and even if it exist, does not depend continuously on the initial data. Therefore, numerical computation is very difficult. We need some special regularization methods to solve this ill-posed problem. Many regularization methods have been proposed before. Such as Fourier regularization method [4], Fourth order modified method [5], Central difference regularization method [6], conjugate gradient method [7], discretization [8], etc.

In this article, we consider the following Cauchy problem in a strip region [9]:

$\begin{cases}u_{x x}+u_{y y}=0, & x \in(0,1), y \in \square, \\ u(0, y)=\varphi(y), & y \in \square, \\ u_{x}(0, y)=0, & y \in \square,\end{cases}$

It is a classical example of ill-posed problems given by Hadamard in his famous paper [10]. We want to seek the

Juanjuan Pu, female, Master, Northwest Normal University , Lanzhou, China.

Xiangtuan Xiong, male, Professor, Doctoral supervisor, Northwest Normal University, Lanzhou, China. solution in the interval $(0,1)$ for problem (1.1). In this article, we will solve the problem (1.1) by a quasi-reversibility regularization method. This regularization method was proposed in reference [11]. And later, it was widely used in reference $[12,13,14,15]$.

To solve the problem (1.1), we need some known facts. Firstly, let $\varphi(\xi)$ denote the Fourier transform of the function $\varphi(y)$ defined by

$$
\varphi(\xi)=\frac{1}{\sqrt{2 \pi}} \int_{-\infty}^{\infty} \varphi(y) e^{-i \xi y} d y,
$$

The corresponding inverse Fourier transform of the function $\varphi(\xi)$ is

$$
\varphi(y)=\frac{1}{\sqrt{2 \pi}} \int_{-\infty}^{\infty} \varphi(\xi) e^{i \xi y} d \xi .
$$

In this paper, $\|\cdot\|$ denote the $L^{2}(\square)$ norm , equivalently, by the Parseval formula

$$
\|\varphi(\cdot)\|=\left(\int_{-\infty}^{\infty}|\varphi(\cdot)|^{2} d \xi\right)^{\frac{1}{2}} .
$$

and we actually have the measured date $\varphi_{\delta}(\cdot)$ satisfy

$$
\left\|\varphi(\cdot)-\varphi_{\delta}(\cdot)\right\| \leq \delta
$$

For problem (1.1), we assume that there exists an a priori bound

$$
\|u(1, \cdot)\| \leq E_{1} .
$$

Now, we assume there is a unique solution of $u(x, y)$, and $\varphi(\cdot), u(x, \cdot) \in L^{2}(\square)$. Application of Fourier transform technique to problem (1.1) with respect to the variable y yields the following problem in a strip region:

$$
\left\{\begin{array}{lc}
u_{x x}(x, \xi)-|\xi|^{2} u(x, \xi)=0, & x \in(0,1), \xi \in \square, \\
u(0, \xi)=\varphi(\xi), & \xi \in \square, \\
u_{x}(0, \xi)=0, & \xi \in \square .
\end{array}\right.
$$

It is easy to see that the solution of problem (1.7) is

$$
u(x, \xi)=\varphi(\xi) \cosh (x|\xi|),
$$

so, the solution of problem (1.1) is given by

$$
u(x, y)=\frac{1}{\sqrt{2 \pi}} \int_{-\infty}^{\infty} \varphi(\xi) \cosh (x|\xi|) e^{i y \xi} d \xi .
$$

Notice that the function $\cosh (x|\xi|) \rightarrow \infty$, as $\xi \rightarrow \infty$.

Therefore, function $\cosh (x|\xi|)$ is unbounded. So, problem (1.1) is severely ill-posed, it is impossible to solve the problem (1.1) by classical method. Then, we will use a new quasi-reversibility regularization method to solve this problem. 


\section{THE QUASI-REVERSIBILITY REGULARIZATION METHOD FOR THE HOMOGENEOUS LAPLACE EQUATION AND ERROR ESTIMATE}

Consider the following problem based on the problem (1.1) in a strip region:

$$
\begin{cases}u_{x x}+u_{y y}=0, & x \in(0,1), y \in \square, \\ u(0, y)+\alpha u(1, y)=\varphi_{\delta}(y), & y \in \square, \\ u_{x}(0, y)=0, & y \in \square .\end{cases}
$$

Where $\alpha$ is regularization parameter, and $0<\alpha<1$, $\delta>0$ denotes the noisy level.

By using the Fourier transform technique to problem (2.1) with respect to the variable $y$ yields the following problem in the frequency space:

$$
\begin{cases}u_{x x}(x, \xi)-|\xi|^{2} u(x, \xi)=0, & x \in(0,1), \xi \in \square, \\ u(0, \xi)+\alpha u(1, \xi)=\varphi_{\delta}(\xi), & \xi \in \square, \\ u_{x}(0, \xi)=0, & \xi \in \square,\end{cases}
$$

The problem (2.2) can be solved as follows:

$$
u(x, \xi)=c_{1} e^{x|\xi|}+c_{2} e^{-x|\xi|},
$$

There is the boundary condition as follows:

$$
\begin{cases}u(0, \xi)+\alpha u(1, \xi)=\varphi_{\delta}(\xi), & \xi \in \square, \\ u_{x}(0, \xi)=0, & \xi \in \square,\end{cases}
$$

Combining (2.3) with (2.4), we get

$$
\begin{cases}c_{1}\left(1+\alpha e^{|\xi|}\right)+c_{2}\left(1+\alpha e^{-|\xi|}\right)=\varphi(\xi), & \xi \in \square, \\ |\xi| c_{1}-|\xi| c_{2}=0, & \xi \in \square,\end{cases}
$$$$
\text { From (2.5), we know } c_{1}=c_{2}=\frac{\varphi_{\delta}(\xi)}{2+\alpha\left(e^{|\xi|}+e^{-|\xi|}\right)} \text {, }
$$

therefore

$$
u(x, \xi)=\frac{\varphi_{\delta}(\xi)}{2+\alpha\left(e^{|\xi|}+e^{-|\xi|}\right)}\left(e^{x|\xi|}+e^{-x|\xi|}\right),
$$

Define the regularization solution of problem (2.2) as follows:

$$
\stackrel{\delta}{u_{\alpha}}(x, \xi)=\frac{2 \varphi_{\delta}(\xi)}{2+\alpha\left(e^{|\xi|}+e^{-|\xi|}\right)} \cosh (x|\xi|) .
$$

By using the inverse Fourier transform technique with respect to the variable $y$, we have

$$
u_{\alpha}^{\delta}(x, y)=\frac{1}{\sqrt{2 \pi}} \int_{-\infty}^{\infty} \frac{2 \varphi_{\delta}(\xi)}{2+\alpha\left(e^{|\xi|}+e^{-|\xi|}\right)} \cosh (x|\xi|) e^{i y \xi} d \xi,
$$

And we have

$$
u_{\alpha}(x, \xi)=\frac{2 \varphi(\xi)}{2+\alpha\left(e^{|\xi|}+e^{-|\xi|}\right)} \cosh (x|\xi|) .
$$

Lemma 2.1. If $0<x<1,0<\alpha<1$, then the following inequality holds

$$
\frac{e^{x|\xi|}}{1+\alpha e^{|\xi|}} \leq \alpha^{-x}(1-x)\left(\frac{x}{1-x}\right)^{x}
$$

Proof. Set $h(|\xi|)=\frac{e^{x|\xi|}}{1+\alpha e^{|\xi|}}$, then

$$
h^{\prime}(|\xi|)=\frac{e^{x|\xi|}\left(x+\alpha x e^{|\xi|}-\alpha e^{|\xi|}\right)}{\left(1+\alpha e^{|\xi|}\right)^{2}}
$$

If $\left|\xi_{0}\right|$ was the zero point of $h^{\prime}(|\xi|)$,then:

$$
\alpha e^{\left|\xi_{0}\right|}=\frac{x}{1-x} \text {, i.e. } e^{x\left|\xi_{0}\right|}=\alpha^{-x}\left(\frac{x}{1-x}\right)^{x},
$$

It is obvious that $\left|\xi_{0}\right|$ is the point of maximum value, then

$$
h(|\xi|)=\frac{e^{x|\xi|}}{1+\alpha e^{|\xi|}} \leq \frac{e^{x\left|\xi_{0}\right|}}{1+\alpha e^{\left|\xi_{0}\right|}}=\alpha^{-x}(1-x)\left(\frac{x}{1-x}\right)^{x} .
$$

Theorem2.1. Let $u(x, y)$ be the solution of problem(1.1) with the exact input data $\varphi(y)$ and $u_{\alpha}^{\delta}(x, y)$ be the regularized solution of (2.8) with the noisy data $\varphi_{\delta}(y)$, If the noisy data $\varphi_{\delta}(y)$ and exact data $\varphi(y)$ satisfy (1.5) and there holds a priori bound (1.6). If the parameter $\alpha$ chosen according to $\alpha=\frac{\delta}{E_{1}}$, then, we have the error estimation:

$$
\left\|u(x, y)-u_{\alpha}^{\delta}(x, y)\right\| \leq 4 C \delta^{1-x} E_{1}{ }^{x} .
$$

Here $C$ is a constant.

Proof. Using Parseval formula and triangle inequality, we have

$$
\begin{aligned}
& \left\|u(x, y)-u_{\alpha}^{\delta}(x, y)\right\| \\
= & \left\|u(x, \xi)-u_{\alpha}^{\delta}(x, \xi)\right\| \\
= & \left\|u(x, \xi)-u_{\alpha}(x, \xi)+u_{\alpha}(x, \xi)-u_{\alpha}^{\delta}(x, \xi)\right\| \\
\leq & \left\|u(x, \xi)-u_{\alpha}(x, \xi)\right\|+\left\|u_{\alpha}(x, \xi)-u_{\alpha}^{\delta}(x, \xi)\right\| \\
: & I_{1}+I_{2} .
\end{aligned}
$$

We first give the estimate of $I_{1}$, combining (1.8), (2.9) with Lemma 2.1, we have

$$
\begin{aligned}
I_{1} & =\left\|u(x, \xi)-u_{\alpha}(x, \xi)\right\| \\
& =\left\|\varphi(\xi) \cosh (x|\xi|)-\frac{2}{2+\alpha\left(e^{|\xi|}+e^{-|\xi|}\right)} \varphi(\xi) \cosh (x|\xi|)\right\| \\
& =\left\|\varphi(\xi) \cosh (x|\xi|)\left(1-\frac{2}{2+\alpha\left(e^{|\xi|}+e^{-|\xi|}\right)}\right)\right\| \\
& =\left\|\varphi(\xi) \cosh (|\xi|) \frac{\cosh (x|\xi|)}{\cosh (|\xi|)} \frac{\alpha\left(e^{|\xi|}+e^{-|\xi|}\right)}{2+\alpha\left(e^{|\xi|}+e^{-|\xi|}\right) \|}\right\| \\
& =\left\|\varphi(\xi) \cosh (|\xi|) \frac{e^{x|\xi|}+e^{-x|\xi|}}{e^{|\xi|}+e^{-|\xi|}} \frac{\alpha\left(e^{|\xi|}+e^{-|\xi|}\right)}{2+\alpha\left(e^{|\xi|}+e^{-|\xi|}\right) \|}\right\| \\
& =\| \varphi(\xi) \cosh (|\xi|) \frac{\alpha\left(e^{x|\xi|}+e^{-x|\xi|}\right)}{2+\alpha\left(e^{|\xi|}+e^{-|\xi|}\right) \|}\left(\frac{x}{x}\right)^{x}, \\
& \leq 2 \alpha E_{1} \sup _{\xi \in \llbracket}\left(\frac{e^{x|\xi|}}{1+\alpha e^{|\xi|}}\right) \leq 2 \alpha^{1-x} E_{1}(1-x)\left(\frac{x}{1-x}\right)^{x}
\end{aligned}
$$

Now we estimate $I_{2}$, combining (2.7), (2.9) with Lemma 2.1, we have 


$$
\begin{aligned}
I_{2} & =\left\|u_{\alpha}(x, \xi)-u_{\alpha}^{\delta}(x, \xi)\right\| \\
& =\| \frac{2 \varphi(\xi) \cosh (x|\xi|)}{2+\alpha\left(e^{|\xi|}+e^{-|\xi|}\right)}-\frac{2 \varphi_{\delta}(\xi) \cosh (x|\xi|)}{2+\alpha\left(e^{|\xi|}+e^{-|\xi|}\right) \|} \\
& =\left\|\frac{2}{2+\alpha\left(e^{|\xi|}+e^{-|\xi|}\right)} \cosh (x|\xi|)\left(\varphi(\xi)-\varphi_{\delta}(\xi)\right)\right\| \\
& =\left\|\frac{2}{2+\alpha\left(e^{|\xi|}+e^{-|\xi|}\right.} \frac{e^{x|\xi|}+e^{-x|\xi|}}{2}\left(\varphi(\xi)-\varphi_{\delta}(\xi)\right)\right\| \\
& \leq 2 \delta \sup _{\xi \in \square}\left(\frac{e^{x|\xi|}}{1+\alpha e^{|\xi|}}\right) \\
& \leq 2 \delta \alpha^{-x}(1-x)\left(\frac{x}{1-x}\right)^{x} .
\end{aligned}
$$

Combining inequality (2.14), (2.15) and (2.16), if select $\alpha=\frac{\delta}{E_{1}}$,estimation (2.13) becomes

$$
\begin{aligned}
& \left\|u(x, y)-u_{\alpha}^{\delta}(x, y)\right\| \\
= & \left\|u(x, \xi)-u_{\alpha}^{\delta}(x, \xi)\right\| \\
= & \left\|u(x, \xi)-u_{\alpha}(x, \xi)+u_{\alpha}(x, \xi)-u_{\alpha}^{\delta}(x, \xi)\right\| \\
\leq & \left\|u(x, \xi)-u_{\alpha}(x, \xi)\right\|+\left\|u_{\alpha}(x, \xi)-u_{\alpha}^{\delta}(x, \xi)\right\| \\
\leq & 2 \alpha^{1-x} E_{1}(1-x)\left(\frac{x}{1-x}\right)^{x}+2 \delta \alpha^{-x}(1-x)\left(\frac{x}{1-x}\right)^{x} \\
\leq & 4 C \delta^{1-x} E_{1}^{x} .
\end{aligned}
$$

Here $C$ is a constant independent of $\delta$ and $E_{1}$.

Now we discuss the error estimate at $x=1$. from (1.8), (2.7) and (2.9), we can obtain

$$
\begin{aligned}
u(1, \xi) & =\varphi(\xi) \cosh (|\xi|), \\
u_{\alpha}(1, \xi) & =\frac{2 \varphi(\xi)}{2+\alpha\left(e^{|\xi|}+e^{-|\xi|}\right)} \cosh (|\xi|), \\
u_{\alpha}^{\delta}(1, \xi) & =\frac{2 \varphi_{\delta}(\xi)}{2+\alpha\left(e^{|\xi|}+e^{-|\xi|}\right)} \cosh (|\xi|),
\end{aligned}
$$

In order to obtain the convergence of error at $x=1$, we give a new a priori assumption:

$$
\left\|u(1, \xi)\left(1+\xi^{2}\right)^{\frac{p}{2}}\right\|_{L^{2}(R)} \leq E_{2},(p>0) .
$$

Theorem2.2. Let $u(1, y)$ be the solution of problem(1.1) at $x=1$ with the exact input data $\varphi(y)$ and $u_{\alpha}^{\delta}(x, y)$ be the regularized solution of (2.8) at $x=1$ with the noisy data $\varphi_{\delta}(y)$, Assume the noisy data $\varphi_{\delta}(y)$ and exact data $\varphi(y)$ satisfy (1.5) and there holds a priori bound (2.21). If the parameter $\alpha$ chosen according to $\alpha=\sqrt{\delta}$,then we have the error estimate:

$$
\left\|u(1, y)-u_{\alpha}^{\delta}(1, y)\right\| \leq \delta^{\frac{1}{2}}+E_{2} \max \left\{2 \delta^{\frac{1}{4}},\left(\frac{2}{\ln \frac{1}{\sqrt{\delta}}}\right)^{p}\right\} .
$$

Proof. Using Parseval formula and triangle inequality, we know

$$
\begin{aligned}
& \left\|u(1, y)-u_{\alpha}^{\delta}(1, y)\right\| \\
= & \left\|u(1, \xi)-u_{\alpha}^{\delta}(1, \xi)\right\| \\
= & \left\|u(1, \xi)-u_{\alpha}(1, \xi)+u_{\alpha}(1, \xi)-u_{\alpha}^{\delta}(1, \xi)\right\| \\
\leq & \left\|u(1, \xi)-u_{\alpha}(1, \xi)\right\|+\left\|u_{\alpha}(1, \xi)-u_{\alpha}^{\delta}(1, \xi)\right\| \\
:= & J_{1}+J_{2} .
\end{aligned}
$$

We first give the estimate of $J_{1}$,combining (2.18),(2.19) with (2.21), we have

$$
\begin{aligned}
& J_{1}=\left\|u(1, \xi)-u_{\alpha}(1, \xi)\right\| \\
& =\left\|\varphi(\xi) \cosh (|\xi|)-\frac{2 \varphi(\xi)}{2+\alpha\left(e^{|\xi|}+e^{-|\xi|}\right)} \cosh (|\xi|)\right\| \\
& =\left\|\varphi(\xi) \cosh (|\xi|)\left(1-\frac{2}{2+\alpha\left(e^{|\xi|}+e^{-|\xi|}\right)}\right)\right\| \\
& =\left\|\frac{\alpha\left(e^{|\xi|}+e^{-|\xi|}\right)}{2+\alpha\left(e^{|\xi|}+e^{-|\xi|}\right)} \varphi(\xi) \cosh (|\xi|)\right\| \| \\
& =\left\|\frac{\alpha\left(e^{|\xi|}+e^{-|\xi|}\right)}{2+\alpha\left(e^{|\xi|}+e^{-|\xi|}\right)}\left(1+\xi^{2}\right)^{-\frac{p}{2}}\left(1+\xi^{2}\right)^{\frac{p}{2}} \varphi(\xi) \cosh (|\xi|)\right\| \\
& \leq E_{2} \sup _{\xi \in \square}\left(\frac{\alpha\left(e^{|\xi|}+e^{-|\xi|}\right)}{2+\alpha\left(e^{|\xi|}+e^{-|\xi|}\right)} \frac{1}{\left(1+\xi^{2}\right)^{\frac{p}{2}}}\right),
\end{aligned}
$$

If we set

$$
f(\xi):=\frac{\alpha\left(e^{|\xi|}+e^{-|\xi|}\right)}{2+\alpha\left(e^{|\xi|}+e^{-|\xi|}\right)} \frac{1}{\left(1+\xi^{2}\right)^{\frac{p}{2}}},
$$

it is easy to see that

$$
\begin{aligned}
& \text { (i) } f(\xi) \leq\left(1+\xi^{2}\right)^{-\frac{p}{2}} \leq|\xi|^{-p} \leq\left(\frac{2}{\ln \frac{1}{\alpha}}\right)^{p}:=\left(\frac{2}{\ln \frac{1}{\sqrt{\delta}}}\right)^{p}, \\
& |\xi| \geq \frac{1}{2} \ln \frac{1}{\alpha} ; \\
& \text { (ii) } f(\xi) \leq 2 \alpha e^{|\xi|} \leq 2 \alpha\left(\frac{1}{\alpha}\right)^{\frac{1}{2}} \leq 2 \alpha^{\frac{1}{2}}:=2 \delta^{\frac{1}{4}},|\xi| \leq \frac{1}{2} \ln \frac{1}{\alpha} .
\end{aligned}
$$

Then we have 


$$
J_{1} \leq E_{2} \max \left\{2 \delta^{\frac{1}{4}},\left(\frac{2}{\ln \frac{1}{\sqrt{\delta}}}\right)^{p}\right\}
$$

Now we estimate $J_{2}$, combining (2.19) with (2.20) we have

$$
\begin{aligned}
J_{2} & =\left\|u_{\alpha}(1, \xi)-u_{\alpha}^{\delta}(1, \xi)\right\| \\
& =\left\|\frac{2 \varphi(\xi)}{2+\alpha\left(e^{|\xi|}+e^{-|\xi|}\right)} \cosh (|\xi|)-\frac{2 \varphi_{\delta}(\xi)}{2+\alpha\left(e^{|\xi|}+e^{-|\xi|}\right)} \cosh (|\xi|)\right\| \\
& =\left\|\frac{2 \cosh (|\xi|)}{2+\alpha\left(e^{|\xi|}+e^{-|\xi|}\right)}\left(\varphi(\xi)-\varphi_{\delta}(\xi)\right)\right\| \\
& =\left\|\frac{\left(e^{|\xi|}+e^{-|\xi|}\right)}{2+\alpha\left(e^{|\xi|}+e^{-|\xi|}\right)}\left(\varphi(\xi)-\varphi_{\delta}(\xi)\right)\right\| \| \\
& \leq \delta \sup _{\xi \in R}\left|\frac{\left(e^{|\xi|}+e^{-|\xi|}\right)}{2+\alpha\left(e^{|\xi|}+e^{-|\xi|}\right)}\right| \leq \frac{\delta}{\alpha},
\end{aligned}
$$

So

$$
J_{2} \leq \delta^{\frac{1}{2}}
$$

Combining inequality (2.23), (2.24) with (2.25), and if select $\alpha=\sqrt{\delta}$,we can obtain

$$
\left\|u(1, y)-u_{\alpha}^{\delta}(1, y)\right\| \leq \delta^{\frac{1}{2}}+E_{2} \max \left\{2 \delta^{\frac{1}{4}},\left(\frac{2}{\ln \frac{1}{\sqrt{\delta}}}\right)^{p}\right\}
$$

\section{THE QUASI-REVERSIBILITY REGULARIZATION METHOD FOR THE NONHOMOGENEOUS LAPLACE EQUATION AND ERROR ESTIMATE}

Consider the following new problem based on the problem (1.1) in a strip region:

$$
\begin{cases}u_{x x}+u_{y y}=f(x, y), & x \in(0,1), y \in \square, \\ u(0, y)=\varphi(y), & y \in \square, \\ u_{x}(0, y)=0, & y \in \square .\end{cases}
$$

By using the Fourier transform technique to problem (3.1) with respect to the variable $y$ yields the following problem in the frequency space

$$
\begin{cases}u_{x x}(x, \xi)-|\xi|^{2} u(x, \xi)=f(x, \xi), & x \in(0,1), \xi \in \square, \\ u(0, \xi)=\varphi(\xi), & \xi \in \square, \\ u_{x}(0, \xi)=0, & \xi \in \square,\end{cases}
$$

The solution to the homogeneous equation of problem (3.2) is

$$
u_{0}(x, \xi)=\varphi(\xi) \cosh (x|\xi|)
$$

Now, set the particular solution to problem (3.2) is

$$
u_{p}=v_{1} u_{1}+v_{2} u_{2} \text {. }
$$

Here $u_{1}=e^{x|\xi|}, u_{2}=e^{-x|\xi|}$ is two basic solution to the homogeneous equation of problem (3.2), then we have

$$
\begin{aligned}
& {\left[\begin{array}{cc}
u_{1} & u_{2} \\
u_{1}^{\prime} & u_{2}^{\prime}
\end{array}\right]\left[\begin{array}{l}
v_{1}^{\prime} \\
v_{2}^{\prime}
\end{array}\right]=\left[\begin{array}{c}
0 \\
f(x, \xi)
\end{array}\right], \text { i.e. }} \\
& {\left[\begin{array}{cc}
e^{x|\xi|} & e^{-x|\xi|} \\
|\xi| e^{x|\xi|} & -|\xi| e^{-x|\xi|}
\end{array}\right]\left[\begin{array}{l}
v_{1}^{\prime} \\
v_{2}^{\prime}
\end{array}\right]=\left[\begin{array}{c}
0 \\
f(x, \xi)
\end{array}\right],}
\end{aligned}
$$

It is easy to see that

$$
\left\{\begin{array}{l}
e^{x|\xi|} v_{1}^{\prime}+e^{-x|\xi|} v_{2}^{\prime}=0 \\
e^{x|\xi|} v_{1}^{\prime}-e^{-x|\xi|} v_{2}^{\prime}=\frac{f(x, \xi)}{|\xi|}
\end{array}\right.
$$

Then, we have

$$
\left\{\begin{array}{l}
v_{1}=\int_{0}^{1} \frac{f(s, \xi)}{2|\xi|} e^{-s|\xi|} d s \\
v_{2}=-\int_{0}^{1} \frac{f(s, \xi)}{2|\xi|} e^{s|\xi|} d s
\end{array}\right.
$$

Combining (3.4) with (3.7), we can obtain

$$
u_{p}=\int_{0}^{1} f(s, \xi) \frac{\sinh [(x-s)|\xi|]}{|\xi|} d s
$$

It is easy to see that

$$
\begin{aligned}
u(x, \xi) & =u_{0}(x, \xi)+u_{p} \\
= & \varphi(\xi) \cosh (x|\xi|)+\int_{0}^{1} f(s, \xi) \frac{\sinh [(x-s)|\xi|]}{|\xi|} d s,
\end{aligned}
$$

By using the inverse Fourier transform technique with respect to the variable $y$, we have

$$
\begin{aligned}
u(x, y)=\frac{1}{\sqrt{2 \pi}} \int_{-\infty}^{\infty} & {[\varphi(\xi) \cosh (x|\xi|)} \\
& \left.+\int_{0}^{1} f(s, \xi) \frac{\sinh [(x-s)|\xi|]}{|\xi|} d s\right] e^{i y \xi} d \xi
\end{aligned}
$$

According to (3.9), there exists

$u(1, \xi)=\varphi(\xi) \cosh (|\xi|)+\int_{0}^{1} f(s, \xi) \frac{\sinh [(1-s)|\xi|]}{|\xi|} d s$

Then

$\varphi(\xi)=\frac{1}{\cosh (|\xi|)}\left(u(1, \xi)+\left(-\int_{0}^{1} f(s, \xi) \frac{\sinh [(1-s)|\xi|]}{|\xi|} d s\right)\right.$

In order to obtain the error estimates, we give the following a priori assumption:

$$
\|u(1, \cdot)\| \leq M .
$$

Lemma 3.1. For $0<s<1$, and $\xi \in \square$, the following inequality hold. 


$$
\frac{\sinh [(1-s)|\xi|]}{|\xi|} \leq e^{(1-s)|\xi|}
$$

Lemma 3.2. Set $A(|\xi|)=-\int_{0}^{1} f(s, \xi) \frac{\sinh [(1-s)|\xi|]}{|\xi|} d s$, and set $\int_{0}^{1}|f(s, \xi)|^{2} d s \leq m_{1} e^{-3|\xi|}$, here $m_{1}$ is a constant, for fixed $s$, and $\xi \in \square$,we have

$$
\begin{aligned}
& \|A(|\xi|)\|^{2}=\left\|-\int_{0}^{1} f(s, \xi) \frac{\sinh [(1-s)|\xi|]}{|\xi|} d s\right\|^{2} \\
& =\int_{-\infty}^{\infty}\left|-\int_{0}^{1} f(s, \xi) \frac{\sinh [(1-s)|\xi|]}{|\xi|} d s\right|^{2} d \xi \\
& \leq \int_{-\infty}^{\infty}\left[\left(\max _{s \in[0,1]} \frac{\sinh [(1-s)|\xi|]}{|\xi|}\right)^{2} \int_{0}^{1}|f(s, \xi)|^{2} d s\right] d \xi \\
& \leq \int_{-\infty}^{\infty} m_{1} e^{2(1-s)|\xi|} e^{-3|\xi|} d \xi \\
& =\int_{-\infty}^{\infty} m_{1} e^{-(1+2 s)|\xi|} d \xi \approx m_{2},
\end{aligned}
$$

Equivalently,

$$
\|A(\xi)\| \leq \sqrt{m_{2}}:=m \text {. }
$$

here $m_{1}, m_{2}, m$ are constant.

Consider the following new problem based on the problem (3.1) in a strip region:

$$
\begin{cases}u_{x x}+u_{y y}=f(x, y), \quad x \in(0,1), & y \in \square, \\ u(0, y)+\alpha u(1, y)=\varphi_{\delta}(y), & y \in \square, \\ u_{x}(0, y)=0, & y \in \square,\end{cases}
$$

By using the Fourier transform technique to problem (3.15) with respect to the variable $y$ yields the following problem in the frequency space:

$$
\begin{cases}u_{x x}(x, \xi)-|\xi|^{2} u(x, \xi)=f(x, \xi), & x \in(0,1), \xi \in \square, \\ u(0, \xi)+\alpha u(1, \xi)=\varphi_{\delta}(\xi), & \xi \in \square, \\ u_{x}(0, \xi)=0, & \xi \in \square,\end{cases}
$$

The solution to the homogeneous equation of problem (3.16) is

$$
u_{0}(x, \xi)=\frac{2 \varphi_{\delta}(\xi)}{2+\alpha\left(e^{|\xi|}+e^{-|\xi|}\right)} \cosh (x|\xi|)
$$

We know the particular solution to problem (3.16) is

$$
u_{p}=\int_{0}^{1} f(s, \xi) \frac{\sinh [(x-s)|\xi|]}{|\xi|} d s
$$

Then, we can obtain the following regularization solution

$$
\begin{aligned}
u_{\alpha}^{\delta}(x, \xi)= & \frac{2 \varphi_{\delta}(\xi)}{2+\alpha\left(e^{|\xi|}+e^{-|\xi|}\right)} \cosh (x|\xi|) \\
& +\int_{0}^{1} f(s, \xi) \frac{\sinh [(x-s)|\xi|]}{|\xi|} d s,
\end{aligned}
$$

and

$$
\begin{aligned}
u_{\alpha}(x, \xi) & =\frac{2 \varphi(\xi)}{2+\alpha\left(e^{|\xi|}+e^{-|\xi|)} \cosh (x|\xi|)\right.} \\
& +\int_{0}^{1} f(s, \xi) \frac{\sinh [(x-s)|\xi|]}{|\xi|} d s,
\end{aligned}
$$

By using the inverse Fourier transform technique with respect to the variable $y$, we have

$$
\begin{aligned}
u_{\alpha}^{\delta}(x, y) & =\frac{1}{\sqrt{2 \pi}} \int_{-\infty}^{\infty}\left[\frac{2 \varphi_{\delta}(\xi)}{2+\alpha\left(e^{|\xi|}+e^{-|\xi|}\right)} \cosh (x|\xi|)\right. \\
& \left.+\int_{0}^{1} f(s, \xi) \frac{\sinh [(x-s)|\xi|]}{|\xi|} d s\right] e^{i y \xi} d \xi
\end{aligned}
$$

Theorem 3.1. Let $u(x, y)$ be the solution of problem(3.1) with the exact input data $\varphi(y)$ and $u_{\alpha}^{\delta}(x, y)$ be the regularized solution of (3.21) with the noisy data $\varphi_{\delta}(y)$ Assume the noisy data $\varphi_{\delta}(y)$ and exact data $\varphi(y)$ and exact satisfy (1.5) and there holds a priori bound (3.13). If the parameter $\alpha$ chosen according to $\alpha=\frac{\delta}{M}$, then we have the error estimate:

$$
\left\|u(x, y)-u_{\alpha}^{\delta}(x, y)\right\| \leq 2 C\left(2+\frac{m}{M}\right) \delta^{1-x} M^{x} .
$$

Proof. using Parseval formula and triangle inequality, we have

$$
\begin{aligned}
& \left\|u(x, y)-u_{\alpha}^{\delta}(x, y)\right\| \\
= & \left\|u(x, \xi)-u_{\alpha}^{\delta}(x, \xi)\right\| \\
= & \left\|u(x, \xi)-u_{\alpha}(x, \xi)+u_{\alpha}(x, \xi)-u_{\alpha}^{\delta}(x, \xi)\right\| \\
\leq & \left\|u(x, \xi)-u_{\alpha}(x, \xi)\right\|+\left\|u_{\alpha}(x, \xi)-u_{\alpha}^{\delta}(x, \xi)\right\| \\
:= & L_{1}+L_{2} .
\end{aligned}
$$

We first give the estimate of $L_{1}$,combining (3.9), (3.12), (3.20), Lemma 2.1 with Lemma 3.2, we have 


$$
\begin{aligned}
L_{1} & =\left\|u(x, \xi)-u_{\alpha}(x, \xi)\right\| \\
& =\left\|\varphi(\xi) \cosh (x|\xi|)-\frac{2 \varphi(\xi)}{2+\alpha\left(e^{|\xi|}+e^{-|\xi|}\right)} \cosh (x|\xi|)\right\| \\
& =\left\|\varphi(\xi) \cosh (x|\xi|)\left(1-\frac{2}{2+\alpha\left(e^{|\xi|}+e^{-|\xi|}\right)}\right)\right\| \\
& =\| \varphi(\xi) \cosh (x|\xi|) \frac{\alpha\left(e^{|\xi|}+e^{-|\xi|}\right)}{2+\alpha\left(e^{|\xi|}+e^{-|\xi|}\right) \|} \\
& =\| \frac{\alpha\left(e^{x|\xi|}+e^{-x|\xi|}\right)}{2+\alpha\left(e^{|\xi|}+e^{-|\xi|}\right)} \cdot \\
& \quad\left(u(1, \xi)+\left(-\int_{0}^{1} f(s, \xi) \frac{\sinh [(1-s)|\xi|]}{|\xi|} d s\right)\right) \| \\
& \quad 2 \alpha \sup \left(\frac{e^{x|\xi|}}{1+\alpha e^{|\xi|}}\right) \cdot\left(M+\left\|\int_{0}^{1} f(s, \xi) \frac{\sinh [(1-s)|\xi|]}{|\xi|} d s\right\| \|\right. \\
& \leq 2(M+m) \alpha^{1-x}(1-x)\left(\frac{x}{1-x}\right)^{x},
\end{aligned}
$$

Now we estimate $L_{2}$,combining (3.19), (3.20) with Lemma 2.1, we have

$$
\begin{aligned}
L_{2} & =\left\|u_{\alpha}(x, \xi)-u_{\alpha}^{\delta}(x, \xi)\right\| \\
& =\| \frac{2 \varphi(\xi) \cosh (x|\xi|)}{2+\alpha\left(e^{|\xi|}+e^{-|\xi|)}\right.}-\frac{2 \varphi_{\delta}(\xi) \cosh (x|\xi|)}{2+\alpha\left(e^{|\xi|}+e^{-|\xi|}\right) \|} \\
& =\left\|\frac{2}{2+\alpha\left(e^{|\xi|}+e^{-|\xi|}\right)} \cosh (x|\xi|)\left(\varphi(\xi)-\varphi_{\delta}(\xi)\right)\right\| \\
& =\left\|\frac{2}{2+\alpha\left(e^{|\xi|}+e^{-|\xi|}\right)} \frac{e^{x|\xi|}+e^{-x|\xi|}}{2}\left(\varphi(\xi)-\varphi_{\delta}(\xi)\right)\right\| \\
& \leq 2 \delta \sup _{\xi \in \llbracket}\left(\frac{e^{x|\xi|}}{1+\alpha e^{|\xi|}}\right) \\
& \leq 2 \delta \alpha^{-x}(1-x)\left(\frac{x}{1-x}\right)^{x} .
\end{aligned}
$$

Combining inequality (3.23), (3.24) with (3.25), if select $\alpha=\frac{\delta}{M}$,estimation (3.22) becomes

$$
\begin{aligned}
& \left\|u(x, y)-u_{\alpha}^{\delta}(x, y)\right\| \\
= & \left\|u(x, \xi)-u_{\alpha}^{\delta}(x, \xi)\right\| \\
= & \left\|u(x, \xi)-u_{\alpha}(x, \xi)+u_{\alpha}(x, \xi)-u_{\alpha}^{\delta}(x, \xi)\right\| \\
\leq & \left\|u(x, \xi)-u_{\alpha}(x, \xi)\right\|+\left\|u_{\alpha}(x, \xi)-u_{\alpha}^{\delta}(x, \xi)\right\| \\
\leq & 2(M+m) \alpha^{1-x}(1-x)\left(\frac{x}{1-x}\right)^{x}+2 \delta \alpha^{-x}(1-x)\left(\frac{x}{1-x}\right)^{x} \\
\leq & 2 C\left(2+\frac{m}{M}\right) \delta^{1-x} M^{x} .
\end{aligned}
$$

Here $m$ and $C$ are constant independent of $\delta$ and $M$.

\section{CONCLUSION}

In this paper, we discuss a Cauchy problem for the homogeneous and nonhomogeneous Laplace equation by a quasi-reversibility regularization method. In the first part, we give a Cauchy problem for Laplace equation in a strip region, and summarize some existing regularization methods to solve this problem. In the second part, the homogeneous Laplace equation is solved by a quasi-reversibility regularization method, and the error estimation with a priori parameter choice rule is obtained. In the third part, the nonhomogeneous Laplace equation is discussed in the same way, and the corresponding error estimate is obtained by the a priori parameter selection rule. This paper only discussed the error estimation under the a priori parameter selection rule. In the following work, we will further discuss the error estimation by the a posteriori parameter choice rules.

\section{REFERENCES}

[1] R. Gorenflo. Funktionentheoretische Bestimmung des Aussenfeldes zu einer zweidimensionalen magnetohydrostatischen Konfiguration[J]. Ztschrift Für Angewandte Mathematik Und Physik Zamp, 1965,16(2):279-290.

[2] P. C. Franzone., E. Magenes.. On the inverse potential problem of electrocardiology[J]. Calcolo, 1979, 16(4):459-538.

[3] C. Vani, A. Avudainayagam. Regularized solution of the Cauchy problem for the Laplace equation using Meyer wavelets[J] Mathematical and Computer Modelling, 2002, 36(9-10):1151-1159.

[4] C. L. Fu, H. Li, Z. Qian, et al. Fourier regularization method for solving a Cauchy problem for the Laplace equation[J]. Inverse Problems in Science and Engineering, 2008,16(2):159-169.

[5] Z. Qian, C. L. Fu, X. T. Xiong. Fourth-order modified method for the Cauchy problem for the Laplace equation[J]. 2006,192(2):205-218.

[6] X.T. Xiong, C. L. Fu. Central difference regularization method for the Cauchy problem of the Laplace's equation[J].2006,181(1):675-684.

[7] D. N. Hào, D. Lesnic. The Cauchy problem for Laplace's equation via the conjugate gradient method[J]. Ima Journal of Applied Mathematics, 2000(2):199-217(19).

[8] H. J. Reinhardt, H. Han, et al. Stability and regularization of a discrete approximation to the Cauchy problem of Laplace equation[J]. Siam Journal on Numerical Analysis, 1999, 36(3):890-905.

[9] D. N. Hào. P. M. Hien. Stability results for the Cauchy problem for the Laplace equation in a strip[J]. Inverse Problems, 2003,19(4):833-840.

[10] J. Hadamard. Lectures on the Cauchy problem in linear partial differential equations, Yale University Press, New Haven, 1923.

[11] M. Denche, K.. Bessila. A modified quasi-boundary value method for ill-posed problems[J]. Journal of Mathematical Analysis and Applications, 2005, 301(2):419-426.

[12] H. W. Zhang. Modified quasi-boundary value method for a Cauchy problem of semi-linear elliptic equation $[\mathrm{J}]$. 
[13] H. Zhang. Modified quasi-boundary value method for Cauchy problems of elliptic equations with variable coefficients[J]. Electronic Journal of Differential Equations, 2011, 2011(106).

[14] ] T. Wei, J. Wang. A modified quasi-boundary value method for an inverse source problem of the time-fractional diffusion equation[J] Applied Numerical Mathematics, 2014, 78:95-111.

[15] F. Yang, Y. Zhang, X. Liu, et al. The Quasi-Boundary Value Method for Identifying the Initial Value of the Space-Time Fractional Diffusion Equation[J]. Acta Mathematica entia, 2020, 40(3):641-658.

Juanjuan Pu, female, Master, Northwest Normal University, Lanzhou, China.

Xiangtuan Xiong, male, Professor, Doctoral supervisor, Northwest Norma University, Lanzhou, China. 